\title{
Rapid Temperature-Dependent Rheological Measurements of Non- Newtonian Solutions Using a Machine-Learning Aided Microfluidic Rheometer
}

\author{
Francesco Del Giudice* and Claire Barnes
}

Cite This: https://doi.org/10.1021/acs.analchem.1c05208

ABSTRACT: Biofluids such as synovial fluid, blood plasma, and saliva contain several proteins which impart non-Newtonian properties to the biofluids. The concentration of such protein macromolecules in biofluids is regarded as an important biomarker for the diagnosis of several health conditions, including cardiovascular disorders, joint quality, and Alzheimer's. Existing technologies for the measurements of macromolecules in biofluids are limited; they require a long turnaround time, or require complex protocols, thus calling for alternative, more suitable, methodologies aimed at such measurements. According to the well-established relations for polymer solutions, the concentration of macromolecules in solutions can also be derived via measure-
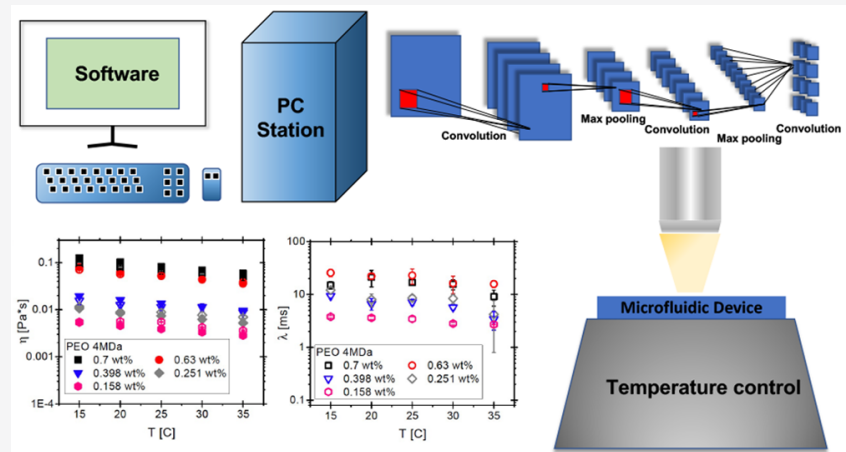
ment of rheological properties such as shear-viscosity and the longest relaxation time. We here introduce a microfluidic rheometer for rapid simultaneous measurement of shear viscosity and longest relaxation time of non-Newtonian solutions at different temperatures. At variance with previous technologies, our microfluidic rheometer provides a very short turnaround time of around 2 min or less thanks to the implementation of a machinelearning algorithm. We validated our platform on several aqueous solutions of poly(ethylene oxide). We also performed measurements on hyaluronic acid solutions in the clinical range for joint grade assessment. We observed monotonic behavior with the concentration for both rheological properties, thus speculating on their use as potential rheo-markers, i.e., rheological biomarkers, across several disease states.

\section{INTRODUCTION}

The concentration of biomacromolecules in biofluids is regarded as an important biomarker across several disease states. For instance, the concentration of hyaluronic acid in synovial fluid can provide an indication regarding the joint grade, ${ }^{1}$ while also offering insights in relation to other conditions such as rheumatoid arthritis ${ }^{2}$ and periprosthetic joint infection. ${ }^{3}$ In the specific case of rheumatoid arthritis, changes in the level of hyaluronic acid have been associated with the inhibition of antithrombin with consequent deterioration of the joint tissue. ${ }^{4}$ Similarly, a decrease in the concentration of hyaluronic acid in synovial fluid has been linked to a pejorative state in rheumatoid arthritis. ${ }^{5}$ For this reason, it is clearly important to monitor the concentration of hyaluronic acid to guide clinical decisions. Synovial fluid is not the only biofluid where the concentration of macromolecules in solution is linked to disease states. An elevated amount of the fibrinogen protein in blood plasma is related to the occurrence and the progression of cardiovascular disorders. ${ }^{6-8}$ There is also some evidence that the concentration of tau protein and amyloid beta in cerebrospinal fluid is linked to the onset of Alzheimer's disease. ${ }^{9}$

There are several techniques to quantify the concentration of macromolecules in biofluids, but they often require either a large amount of sample or a turnaround time of up to several hours. Furthermore, some of the most common protein assays require several steps to be carried out by an operator familiar with protein handling together with access to specialized equipment. ${ }^{10-12}$ Another potential way of measuring protein concentration is via indirect methodologies. The majority of biofluids of interest in medical applications, including cerebrospinal fluid, blood plasma, and synovial fluid, are mostly made of water, which is a Newtonian fluid, meaning that its viscosity does not change with the external flow

Received: December 1, 2021

Accepted: February 7, 2022 
conditions. The addition of protein macromolecules to water, however, imparts additional viscous and elastic components to the fluid, thus making the resulting solution non-Newtonian. Typical examples of non-Newtonian biofluids include blood plasma, ${ }^{13,14}$ saliva, ${ }^{15}$ and synovial fluid. ${ }^{16}$ According to the well-established relations in polymer physics, ${ }^{17,18}$ the concentration of macromolecules in solution can be extrapolated via the measurement of rheological properties such as shear viscosity and longest relaxation time. The shear viscosity is a measure of the drag exerted by the biofluid when subjected to an external flow, while the longest relaxation time is a measure of the elasticity stored within the macromolecules (or proteins) suspended in the solution. This concept has been previously employed to introduce the shear viscosity as a potential biomarker for several diseases, including cardiovascular diseases, $^{6-8}$ and periprosthetic joint infections. ${ }^{3}$ The measurement of the shear viscosity, however, has always been affected by some limitations related to the piece of equipment employed for such measurement. The conventional rheometer, considered the gold standard for rheological analysis, requires sample sizes of several milliliters to perform the measurement and requires a long waiting time to obtain results. More importantly, measuring protein solutions with a conventional rheometer can lead to measurement artifacts caused by the aggregation of proteins at the edge of the geometry employed for the measurement. ${ }^{19}$ Finally, conventional rheometers are not sensitive enough to measure the longest relaxation time of a dilute concentration of macromolecules, a typical condition for biofluids. ${ }^{20}$ The longest relaxation time is an important property as it displays a stronger dependence on the molecular weight than the shear viscosity, ${ }^{18}$ especially in dilute and semidilute unentangled systems, thus making it a potential biomarker to identify the contribution of large macromolecules over smaller ones in solution. This is especially important in the clinical context: being able to single out, for instance, the contribution of the hyaluronic acid molecules having larger molecular weight in synovial fluid (which is made of hyaluronic acid molecules having different molecular weights) can provide accurate information regarding the joint grade. ${ }^{1}$ Similarly, fibrinogen is generally the macromolecule with larger molecular weight in blood plasma: since its concentration in the ensemble of other proteins in blood plasma is related to cardiovascular events, ${ }^{6-8}$ the longest relaxation time value of the blood plasma would be affected largely by the fibrinogen rather than other molecules.

The requirement of measuring the rheological properties of biofluids has prompted significant research in the field of microfluidic rheometry, ${ }^{16,20,21}$ i.e., microfluidic technologies to measure rheological properties. Microfluidic technologies have the advantage of requiring a small amount of sample. They can easily be integrated with other platforms, and they are generally in a closed-geometry (e.g., closed channels), meaning that measurement artifacts due to edge effects can be avoided: these conditions are highly sought for biofluid characterization. ${ }^{20}$ Choi and Park ${ }^{22}$ introduced a microfluidic device for the measurements of the shear viscosity by direct comparison of the flow within the microfluidic device with the flow of a reference fluid. They also demonstrated the possibility of performing measurements at different temperatures by fitting the whole experimental apparatus in an incubator. Arosio et al. $^{23}$ introduced a different microfluidic device to measure the viscosity based on the advection and the diffusion of particles suspended into the solutions under investigation. Lee et al. ${ }^{24}$ introduced a microfluidic rheometer for the measurements of shear viscosity at different temperatures. Kang et al. ${ }^{25}$ introduced a paper-based microfluidic viscometer to measure the viscosity of blood plasma using a colorimetric approach. Several other microfluidic techniques have been introduced, and we invite the interested reader to look at existing reviews $^{16,20,21}$ and the references therein. The technologies introduced so far often required complex channel designs or operations, and their applicability was limited to a few polymer solutions. Furthermore, such microfluidic devices have only been able to measure one property, the shear viscosity, while neglecting other important rheological properties such as the longest relaxation time. This problem has only been partially addressed with the recent introduction of the $\mu$-rheometer, ${ }^{26}$ which allowed the simultaneous measurement of shear viscosity and the longest relaxation time in the same microfluidic device. The $\mu$-rheometer was an extension of the original one developed for the measurement of the longest relaxation time only. ${ }^{27,28}$ However, the $\mu$-rheometer suffered from two important drawbacks: first, the working principle was based on performing particle tracking of flowing particles, a process that could take several minutes or several iteration steps to improve accuracy; second, only measurements at laboratory temperature could be performed, thus limiting its potential applications in the biomedical field, where measurements are often carried out at the physiological temperature of $T \simeq 37{ }^{\circ} \mathrm{C}$.

To address these challenges, we here introduce a microfluidic rheometer for rapid temperature-dependent rheological measurement of non-Newtonian solutions. The novelty of our work is the integration of a particle tracking machine learning algorithm to a microfluidic rheometer functioning according to the working principle of the $\mu$-rheometer introduced earlier. ${ }^{26}$ Thanks to such integration, the time required for data processing was reduced to less than a minute, allowing rapid temperature-dependent rheological characterization of nonNewtonian solutions in a microfluidic device using between 100 and $200 \mu \mathrm{L}$ of samples only, thus making it an ideal platform for the characterization of biofluids within the clinical context. We first demonstrated the accuracy of our microfluidic rheometer by comparing the measured rheological parameters with those derived via conventional rheology techniques on several aqueous solutions of poly(ethylene oxide) (PEO). We also repeated the measurements on hyaluronic acid (HA) solutions at concentrations comparable with the clinical range for joint grade, ${ }^{1}$ observing that both shear viscosity $\eta$ and longest relaxation time $\lambda$ varied monotonically with the mass concentration in the whole temperature range investigated, thus innovatively providing a proof of concept for the fact that $\eta$ and $\lambda$ can both be used as rheomarkers, i.e., rheological biomarkers, for the diagnosis of several disease states. Finally, we also designed a simple MATLAB application to rapidly process the videos obtained using the microfluidic rheometer and to then obtain both rheological and flow parameters.

\section{MATERIALS AND METHODS}

2.1. Material and Preparation. Two different sets of nonNewtonian polymer solutions, poly(ethylene oxide) (PEO) and hyaluronic acid (HA), were employed. Aqueous solutions of PEO (Sigma-Aldrich UK) with molecular weight $M_{\mathrm{w}}=4$ $\mathrm{MDa}$ at different mass concentrations in the range of $0.158<c$ $<0.7$ wt $\%$ were prepared. A PEO solution at concentration of $c=0.7$ wt $\%$ was prepared by direct addition of the polymer 

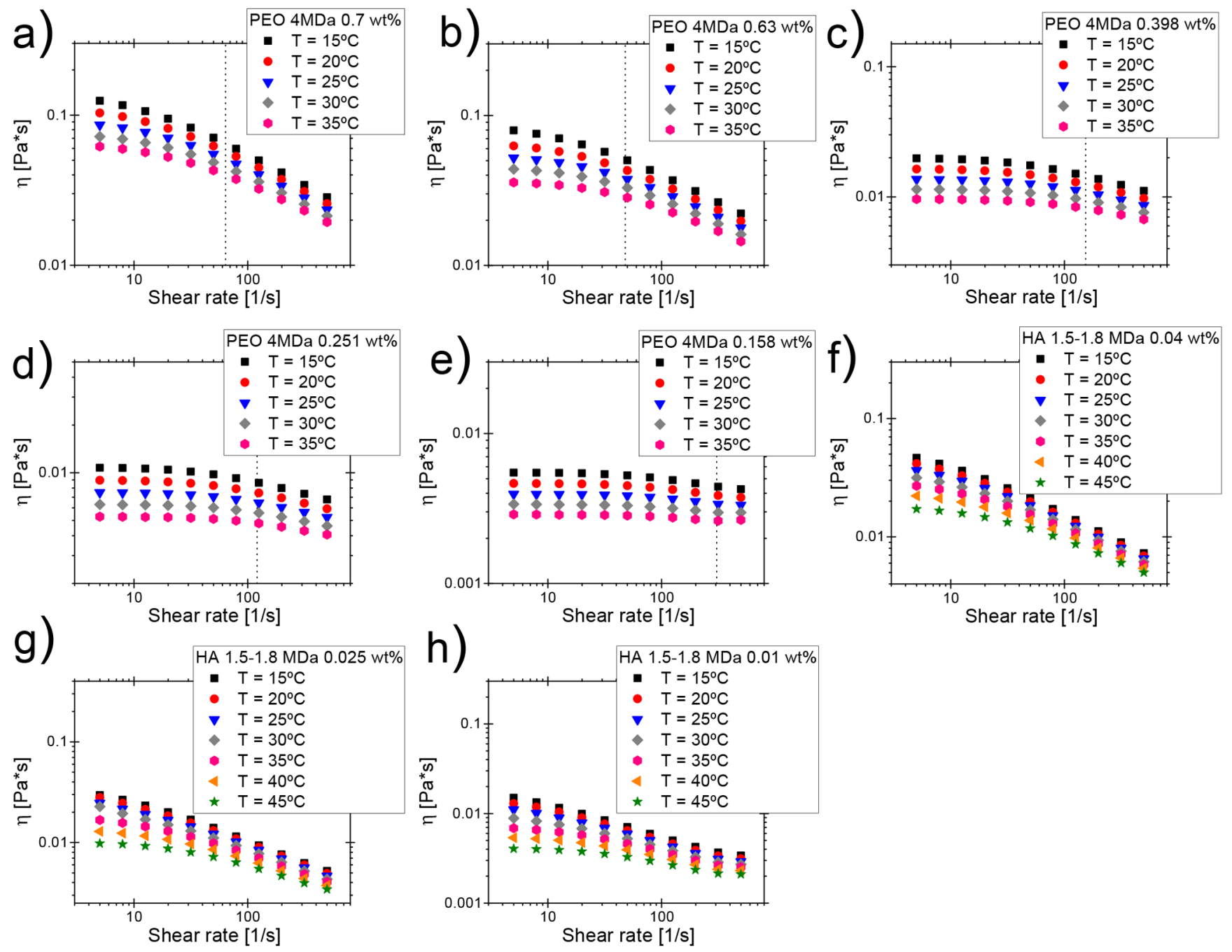

Figure 1. (a-e) Shear viscosity as a function of the shear rate for different PEO solutions in the temperature range of $15<T<35^{\circ} \mathrm{C}$. Depending on the polymer concentration, the solutions displayed either mild or negligible shear-thinning. The vertical dashed dots represent the shear rate value $\dot{\gamma}$ calculated as $\dot{\gamma}=1 / \lambda$, where $\lambda$ is the longest relaxation time evaluated using the microfluidic rheometer, with data reported in Figure 5 . (fh) Shear viscosity as a function of the shear rate for different HA solutions in the temperature range of $15<T<45{ }^{\circ} \mathrm{C}$.

powder. The other solutions were prepared by dilution of the stock, using Gilson pipettes and a scale with $0.1 \mathrm{mg}$ precision (Ohaus Adventurer Precision Balances). Aqueous solutions of HA (Sigma-Aldrich UK) with molecular weight $M_{\mathrm{w}}=1.5-1.8$ $\mathrm{MDa}$ were prepared at mass concentrations of $0.01,0.025$, and 0.04 wt $\%$. These concentrations for HA were chosen because they were in line with the concentration of HA found in synovial fluid for different joint states. ${ }^{1} \mathrm{~A} \mathrm{HA}$ solution at a concentration of $c=0.04$ wt $\%$ was prepared by direct addition of the polymer powder. The other solutions were prepared by dilution of the stock, as described previously.

Our rheological measurements using the microfluidic rheometer required the addition of particles to the solutions of interest (see section 3 for more details). Polystyrene particles (Polysciences Inc.) with density $\rho_{\mathrm{p}}=1.05 \mathrm{~g} / \mathrm{L}$ and diameters $d_{\mathrm{p}}=10 \pm 1 \mu \mathrm{m}$ were added to the solutions. Dilute suspensions with volume fraction $\phi=0.01 \mathrm{wt} \%$ were prepared by direct addition of particles to the polymer solution, and by using a mixer (Fisher Scientific). The effect of the particle addition on the fluid rheology can be neglected at such small $\phi$ values. $^{29}$
2.2. Bulk Shear Rheometry. The viscosity curves for all of the polymer solutions investigated were evaluated using a stress-controlled TA Instruments HR-30 rheometer. A metal cone with a $60 \mathrm{~mm}$ diameter and cone angle of $2^{\circ}$ was used. The temperature was controlled via a Peltier system, in the range $15<T<45^{\circ} \mathrm{C}$. For the PEO solutions, we carried out conventional rheology measurements in order to derive the viscosity curve, represented as shear viscosity $\eta$ plotted as a function of the shear rate $\dot{\gamma}$ (Figure $1 \mathrm{a}-\mathrm{e}$ ). At a PEO mass concentration of $c=0.7$ wt $\%$ and of $c=0.63 \mathrm{wt} \%$, the viscosity exhibited a mild shear-thinning behavior above a shear-rate value of $\dot{\gamma} \approx 10 \mathrm{~s}^{-1}$. When decreasing the concentration further, the shear-thinning became negligible. Regardless of the mass concentration, the shear viscosity decreased monotonically when increasing the temperature, in good agreement with previous findings in the same temperature range. ${ }^{30}$ We then repeated the same procedure, and we measured the viscosity curve for the three HA concentrations employed here (in the clinical range for assessing joint quality ${ }^{1}$ ), finding a rheological trend similar to the PEO solutions. We observed that all of the solutions exhibited a mild shear-thinning behavior (Figure 1f-h). 


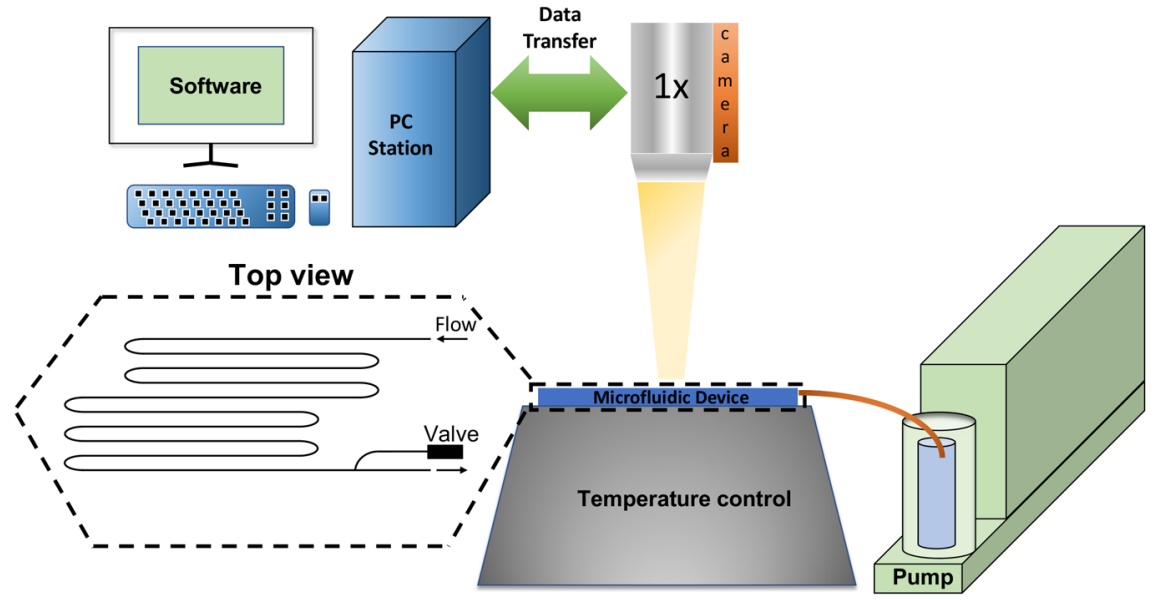

Figure 2. Schematic of the microfluidic station employed in this work. The glass vial contains the fluid to characterize. The pump controls the flow inside the microfluidic device. The temperature in the microfluidic device is controlled using a temperature control station with a metallic plate on top; the microfluidic device is positioned on the metal part so that heat transfer can occur efficiently. Image acquisition is carried out using a reflective digital microscope with a fast camera embedded in it. The videos of flowing particles are transferred to the computer and subsequently analyzed. Dimensions are not to scale.

2.3. Microfluidic Setup. We employed the microfluidic apparatus schematized in Figure 2. The non-Newtonian solutions employed in this work were pumped in the microfluidic device using a pressure pump (p-pump Dolomite Microfluidics) connected to a compressor (Balbi instrument) acting as an air-supplier. The microfluidic device was a commercial serpentine $\mathrm{T}$-junction glass hydrophilic device (Dolomite Microfluidics) with a nearly circular cross-section having a diameter $D=100 \mu \mathrm{m}$, and where the short junction was closed using a microfluidic valve (Dolomite Microfluidics) to obtain a one-inlet-one-outlet device (top view in Figure 2). The flow in the microfluidic device was observed using a Meros reflective high-speed digital microscope (Dolomite Microfluidics). Videos were acquired using the Meros digital microscope at frame rate values between 50 and $200 \mathrm{fps}$, depending on the imposed pressure drop in the range $100<$ $\Delta P<400 \mathrm{mbar}$. The exposure time was fixed to $0.05 \mathrm{~ms}$. The temperature in the microfluidic device was controlled in the range $15<T<45{ }^{\circ} \mathrm{C}$ using the Meros temperature control unit (Dolomite Microfluidics), which can control the temperature in the range 0 to $100{ }^{\circ} \mathrm{C}$ with a $0.1{ }^{\circ} \mathrm{C}$ accuracy. The Meros temperature unit features a metal plate on the top where the temperature is equal to the imposed temperature. The glass microfluidic device was in direct contact with the metal plate (Figure 2), meaning that the temperature within the microfluidic device was equal to the imposed temperature. All of the devices were controlled using the Dolomite Software. For each polymer concentration and for each temperature, we performed three independent measurements spaced a few minutes apart, noticing no difference at all in the particle velocity, meaning that the system had achieved a steady state. The rheological properties were subsequently evaluated as the average of the three experiments. Data analysis was carried out on a Deep Learning unit (3XS Deep Learning DBP C420C, Scan), with a 24 GB GDDR6X Graphics Card, DDR4 2666 $\mathrm{MHz}$ Memory Kit $8 \times 16 \mathrm{~GB}$, Intel 10 Core i9 $10900 \mathrm{X}$ Unlocked Cascade Lake-X CPU/Processor, and ASUS Intel Core-X WS X299 SAGE II Dual 2.5G LAN CEB Workstation Motherboard. Please note that the data analysis to derive both shear viscosity and the longest relaxation time can be carried out on any type of computer or laptop, not necessarily on the one described here.

\section{WORKING PRINCIPLE OF THE MICROFLUIDIC RHEOMETER}

3.1. Summary of the Theory and the Equations. The working principle of the microfluidic rheometer presented here (hereafter, simply, the microfluidic rheometer) is very similar to the $\mu$-rheometer introduced previously, ${ }^{26}$ and it is based on the well-established phenomenon of viscoelastic transversal migration of particles flowing in a microchannel..$^{31-33}$ We now proceed to briefly summarize the working principle, while the interested reader can find more details in the original publication ${ }^{26}$ and in the references therein.

The microfluidic rheometer allows simultaneous measurement of shear viscosity and the longest relaxation time when the non-Newtonian fluid presents negligible shear-thinning features, i.e., is elastic but with a near constant viscosity over a wide range of shear rates. This is often the case for linear macromolecules at mass concentrations falling in the dilute and semidilute unentangled regime. For fluids presenting shear-thinning features, the measurement is valid as long as the overall shear rate $\dot{\gamma}$ in the channel, defined as $\dot{\gamma}=\bar{v} / D$, where $\bar{v}$ is the average velocity in the channel and $D$ is the channel diameter, falls below the onset of the shear thinning. Rigid particles are first added to the polymer solutions that need to be investigated, and the resulting suspension is pumped inside a microfluidic device having a single-inlet, single-outlet structure (e.g., a straight capillary or a serpentine microchannel like the one employed in this work). Because of the Poiseuillelike flow inside the microchannel ${ }^{34}$ (i.e., parabolic velocity profile), viscoelastic forces act on the suspended particles and lead to a cross-stream migration toward the channel centerline when the suspending fluid either presents a near constant viscosity or negligible shear-thinning properties. ${ }^{31,35,36}$ Particles flowing at the centerline display a velocity which is very close to the centerline streamline fluid velocity when the confinement ratio $\beta$ defined as particle diameter $d_{\mathrm{p}}$ over channel diameter $D$ is $^{37} \beta=d_{\mathrm{p}} / D \approx 0.1$. In other words, when 


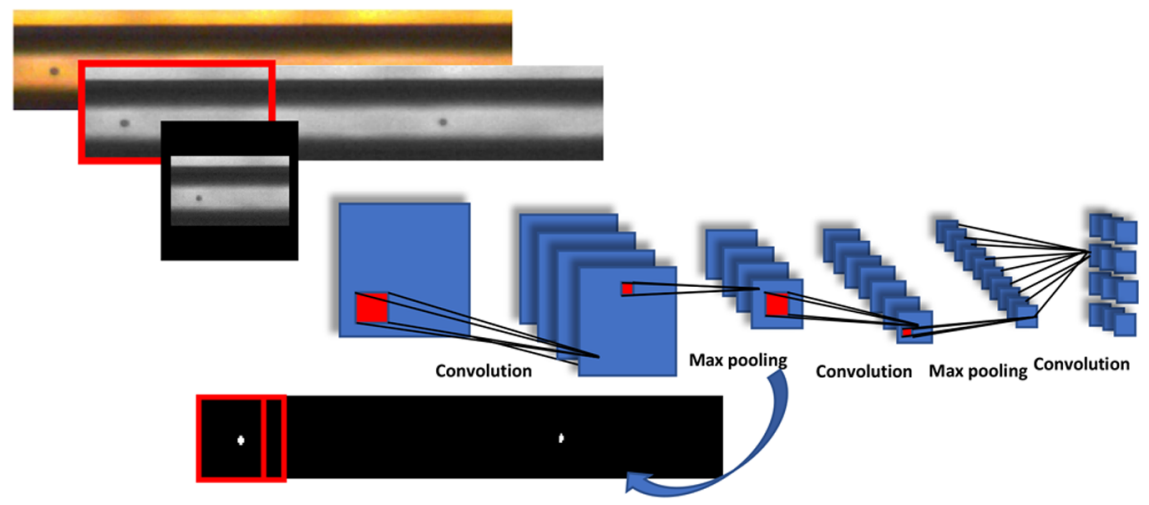

Figure 3. Schematic of our particle tracking algorithm. Videos fed into the software as avi files are converted into greyscale images. A small section of the image is extracted and presented to the fully trained network. The convolutional network is trained to predict a number of outputs for each image including lighting and magnification. On the basis of the predictions made by the network, a bounding box is sized, and a threshold is set to analyze and track particles from a binary image based on thresholds set as a result of the estimate of lighting conditions made by the network.

$\beta=d_{\mathrm{p}} / D \approx 0.1$, the flow disturbance caused by the particle can be neglected.

Using the microfluidic rheometer, the shear viscosity $\eta$ can be evaluated using the Hagen-Poiseuille law: ${ }^{26}$

$$
\eta=\frac{\Delta P}{\mathcal{R Q}}
$$

where $\Delta P$ is the imposed pressure drop, $Q$ is the volumetric flow rate, and $\mathcal{R}$ is the flow resistance which depends upon the geometry (e.g., channel dimensions, cross-section, and length) of the microfluidic device. The flow resistance can be calibrated using a Newtonian liquid such as water, while the volumetric flow rate of non-Newtonian flowing fluids with negligible shear-thinning features can be calculated using the velocity of the particles flowing at the centerline as ${ }^{26,34} Q=$ $v_{\max } / 2 \times \pi D^{2} / 4$, where $v_{\max }$ is the maximum velocity of the fluid, i.e., at the centerline. When $\beta \leq 0.1$ and particles are flowing at the centerline because of the viscoelastic transversal migration, it is possible to make the approximation ${ }^{37} v_{\max } \approx v_{\mathrm{p}}$, where $v_{\mathrm{p}}$ is the particle velocity. The volumetric flow rate can then be calculated as $Q=v_{\mathrm{p}} \pi D^{2} / 8$. Particle velocity $v_{\mathrm{p}}$ is derived via particle tracking. Since the value of the pressure drop $\Delta P$ is known, and $\mathcal{R}$ can be calibrated, the viscosity can be directly calculated using eq 1 .

The longest relaxation time can be calculated using the expression below: ${ }^{26}$

$$
\lambda=\frac{\pi}{4} \frac{1}{\beta^{2}} \frac{D^{4}}{L_{z} Q} \sqrt{\frac{1}{C} \ln \left(\frac{f_{1} B}{1-f_{1}}\right)}
$$

where $L_{z}$ is the distance from the channel inlet, $B=2.7$ and $C$ $=2.75$ are constants derived from the best fit of the theoretical model employed to describe the transversal particle migration phenomenon, ${ }^{27,38}$ and $f_{1}$ is the normalized fraction of particles aligned on the channel centerline.

The validity of eq 2 is constrained by a few requirements discussed below (full details are in the original publications). ${ }^{26,27}$ Inertial effects, quantified via the Reynolds number $R e=\rho \bar{\nu} D / \eta$ should be negligible, meaning $R e<1$. Also, the non-Newtonian fluid should span the linear viscoelastic regime, meaning that the Deborah number, defined as $D e=\lambda \dot{\gamma}$ should be $D e<1$. The confinement ratio should be $\beta \approx 0.1$. Finally, the parameter $\Theta=\operatorname{De}\left(L_{z} / D\right) \beta^{2}$ should be $\Theta<1.4$, or the longest relaxation time may be misestimated. ${ }^{26,27}$

If the requirements above are fulfilled, particle tracking is then used to evaluate the particle velocities and, consequently, the normalized fraction of particles $f_{1}$. From the particle velocity, the volumetric flow rate $Q$ can also be evaluated: once these two values are known, the shear viscosity and the longest relaxation time can be calculated simultaneously using eq 1 and eq 2 , respectively.

At variance with previous works, ${ }^{26,27}$ we here employed machine learning techniques to complete the particle tracking phenomenon in less than a minute (full details in section 3.2). This is very important, as the two assumptions discussed above for the validity of the values obtained using the microfluidic rheometer, i.e., $D e<1$ and $\Theta<1.4$, both depend upon the longest relaxation time $\lambda$. Since $\lambda$ is measured via eq 2 , the two parameters $D e$ and $\Theta$ can only be evaluated after the measurement has been completed. Thanks to the implementation of the machine learning for rapid particle tracking, we could adjust almost in real-time the experimental parameters in order to fulfill the requirements of the theoretical model, thus overcoming one of the main limitations of the previous $\mu$ rheometer. $^{26}$

3.2. Machine-Learning-Based Particle Tracking. The ability to determine the trajectory of particles in a channel has a number of important applications, and therefore single particle and cell tracking is a well-studied area that has led to a number of methods and tools to describe particle behavior, including various machine learning approaches. ${ }^{39-41}$ Often, these solutions are highly specific to a particular application, and as a result, there is no clear way of determining the right algorithm for any given data set. In recent times, there has been a call for researchers to make their methods easily accessible through straightforward installations and an intuitive user interface. ${ }^{39}$ Moreover, the issues around selecting correct solutions for particular applications could be minimized by tools that are highly flexible and are based on fewer prior assumptions. It is important to note that while maximum flexibility is not an immediate consideration for the application presented in this work, such additional capability would make a number of future applications possible. Additionally, a fully flexible system is expected to address several common issues created by varying the experimental apparatus, training methods, and image quality. Existing studies had fallen largely 
into two categories: (i) tools employing largely iterative algorithms to track particles ${ }^{42,43}$ and (ii) computational solutions that have used a machine or deep learning perspective to track particles through space and time. ${ }^{4,45}$ Here, we brought together these two approaches using an iterative algorithm to track particles frame-by-frame along with deep learning methods to execute adequate preprocessing of images, improve accuracy, and ensure the flexibility of our software. Our method is similar to those employed by some of the most recent object detector algorithms, ${ }^{46}$ able to classify and track objects through the use of neural networks. At variance with existing methods, and to reduce the time required for particle tracking, we utilized the above-mentioned algorithm technique ${ }^{46}$ just once at the very start of our algorithm to provide key information to inform tracking.

The particles in this application, though representing just a small number of pixels on an even lighter background, lent themselves to the application of binarization with little amount of background noise or artifacts to account for. Our algorithm employed Otsu's method of binarization ${ }^{47}$ with a threshold set by our convolutional neural network to minimize noise (as schematized in Figure 3). Artificial variation was also introduced by the production of images under poor lighting conditions; the neural network successfully recognized the poorer contrast conditions and was able to adjust the settings accordingly. Upon binarization, some basic statistics could be gathered from each image and particles could be isolated based on size and circularity, set according to the initial assessment of the images by the network. A key challenge associated with these images was the similarity of particles in terms of their size and general appearance, which made distinguishing these fast moving objects in close proximity from one another to enable successful tracking through consecutive frames an important consideration. Once images were in the correct format, we employed the use of a relatively small analyzing window termed a bounding box (highlighted in red in Figure 3). The dimensions of our bounding box were based on parameters provided by the network's initial assessment: this approach allowed us to implement a set of assumptions and rules to successfully track the particles by dealing with just a few at any given time. A spatial threshold was set to signal that a particle had come near the end of the analyzing window and initiated the software to step back through time to track the particle over the previous three to four frames. In order to correctly link particles between each frame, the following simple rules were used (assuming that the article moved only along the flow direction in the experimental window):

$$
X_{i, t-1}=\max \left\{X_{j, t-1}\right\} \text { for all } j \in N \text { given that } X_{i, t-1} \leq X_{i, t}
$$

where $X$ is the particle position along the flow direction, $N$ is the total number of particles in an analyzing window at any given time point, and $t$ is the time point under consideration. Once a particle was tracked back through time, evaluation of its position at any given point could be determined based on image statistics taken from the images through time, and therefore, estimates of each particle's velocity could also be made. Thanks to the simplistic nature of our particle trajectories, the small bounding box did not compromise the accuracy, which may be a concern. ${ }^{39}$ It is important to note that, as videos were being processed, our software was dynamically improving in terms of performance, completing some spot checks for noise in the images and particles that dropped out frame to frame due to large variations in intensity and poor image quality. These images could then be used to improve the overall software and retrain the networks. Such an approach led to improved accuracy and efficiency of our algorithm. The velocities of all particles estimated by the software were compared with estimates provided using a wellestablished IDL software algorithm. ${ }^{48}$ Our software showed excellent agreement with the IDL values, despite taking a fraction of the time (data not included).

\section{RESULTS AND DISCUSSION}

4.1. Calibration of the Microfluidic Rheometer. The first step required to perform measurements using the microfluidic rheometer was the calibration of the geometrical resistance $\mathcal{R}$ in eq 1 . The resistance $\mathcal{R}$ depends entirely on the geometry of the channel, while not depending on other factors such as volumetric flow rate $Q$ pressure drop $\Delta P$ or temperature $T$. We then performed the calibration using deionized water (a Newtonian liquid), according to the following procedure. We employed the apparatus schematized in Figure 2 with the addition of a flow sensor (Dolomite Microfluidics) to read the volumetric flow rate. For each pressure drop, we then computed the volumetric flow rate for three values of imposed temperature, namely, 10, 20, and 35 ${ }^{\circ} \mathrm{C}$, while the room temperature was $T=20{ }^{\circ} \mathrm{C}$. The geometrical resistance was calculated by rearranging eq 1 , as $\mathcal{R}=\Delta P /(Q \eta)$, where the viscosity of water at the three temperatures was taken from the Anton Paar Web site (https://wiki.anton-paar.com/uk-en/water/). We then plotted $\Delta P$ as a function of $Q \eta$, so that the slope would return the value of the flow resistance $\mathcal{R}$ (Figure 4 ). The data followed a

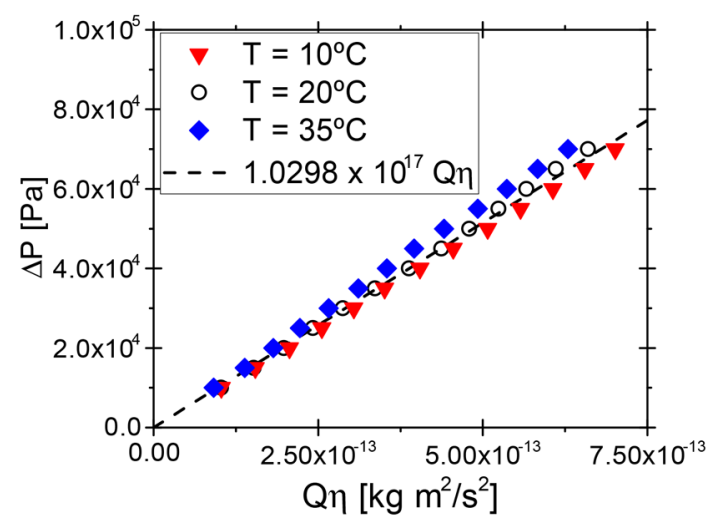

Figure 4. Calibration of the microfluidic rheometer using deionized water (Newtonian liquid). The imposed pressure drop $\Delta P$ is plotted against the product of the volumetric flow rate $Q$ and of the shear viscosity $\eta$ for three different temperatures. The dashed line is the best linear fit of the data at $T=20{ }^{\circ} \mathrm{C}$. The slope of the dashed line provides the value of the geometric resistance, calculated by rearranging eq 1 , as $\mathcal{R}=\Delta P /(Q \eta)$.

straight line regardless of the temperature; however, they displayed slightly different slopes. We ascribed such minor discrepancy (within 10\%) to the fact that the sample reached a constant temperature equal to the one imposed via the control unit only when flowing within the microfluidic channel; when, instead, the fluid was either in the reservoir or was flowing inside the connecting tubes, the fluid temperature was equal to the laboratory temperature (on average between 18 and 20 ${ }^{\circ} \mathrm{C}$ ). This meant that while the fluid flowing in the device was 
a)

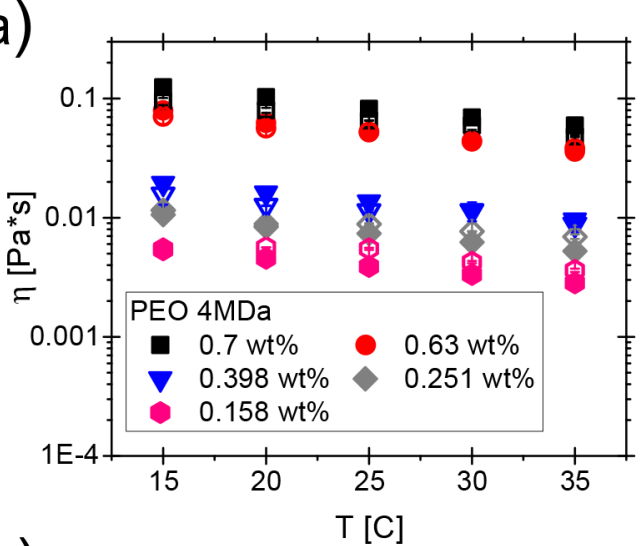

c)

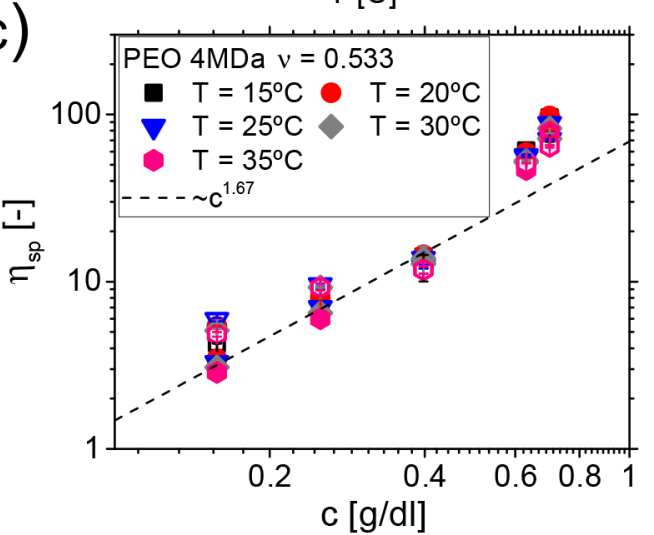

e)

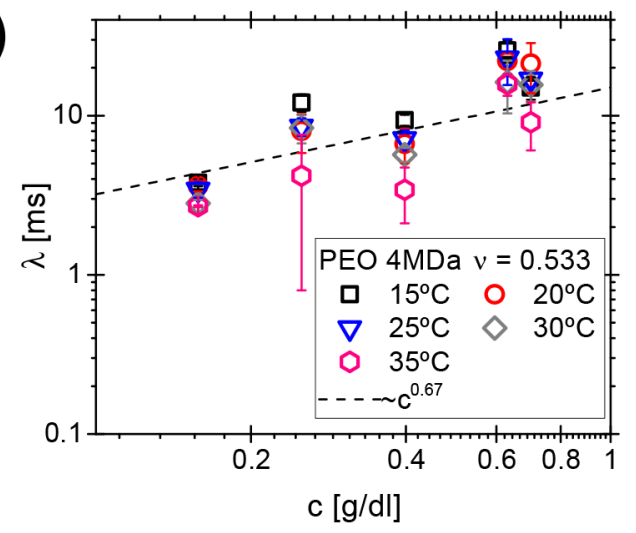

b)

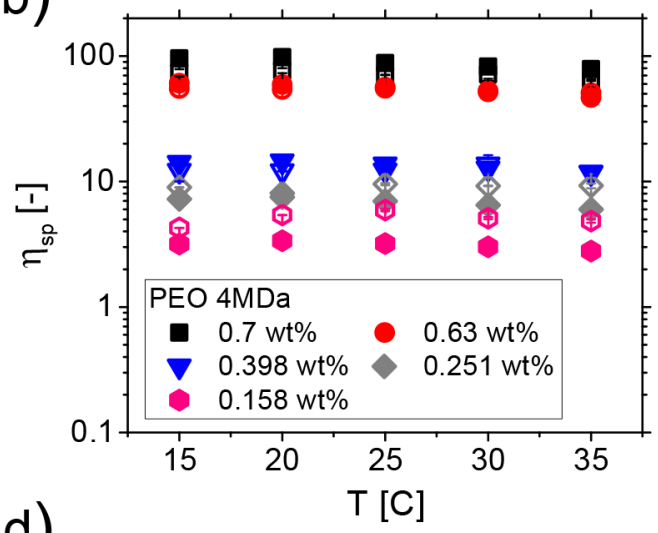

d)

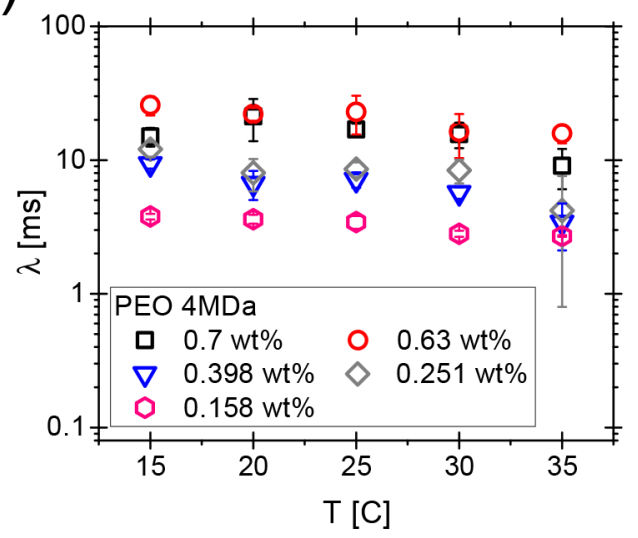

Figure 5. Good agreement is found between conventional rheology and microfluidic rheometry data for poly(ethylene oxide) (PEO) aqueous solutions. (a) Comparison between conventional rheometry data (closed symbols) and microfluidic rheometry data (open symbols) for the viscosity as a function of temperature, for all of the PEO solutions investigated in this work. Values of the viscosity were compared at the same shear rate. Very good agreement was observed for all of the solutions. (b) Same data as in panel a, plotted in terms of specific viscosity $\eta_{\mathrm{sp}}=\eta / \eta_{\mathrm{s}}-1$, where $\eta_{\mathrm{s}}$ is the solvent viscosity (water in this case). (c) Data of panel b, plotted as a function of the mass concentration. (d) Longest relaxation time $\lambda$ measured using the microfluidic rheometer as a function of the temperature $T$ for all of the PEO solutions investigated. (e) Data as in panel $\mathrm{d}$ plotted as a function of the mass concentration $c$. Dashed lines in panels $\mathrm{c}$ and e are theoretical predictions for polymers in relatively good solvent. $^{18}$

at a certain controlled temperature, meaning at a certain viscosity, the fluid in the reservoir and the connecting tubes was at laboratory temperature, meaning at a different viscosity: since we used eq 1 to derive a single value of the fluid viscosity calculated at the temperature controlled via the temperature unit, a minor deviation from the data at $T=20{ }^{\circ} \mathrm{C}$ was to be expected. Data at $T=20^{\circ} \mathrm{C}$ were the best to evaluate the value of the flow resistance, the room temperature being near $T=20$ ${ }^{\circ} \mathrm{C}$; a fitting of this set of data returned a value of flow resistance of $\mathcal{R}=1.0298 \times 10^{17} \mathrm{~m}^{-3}$. This value was kept constant during the data analysis.
4.2. Device Accuracy Using Poly(ethylene oxide) Solutions. In this section, we demonstrate the accuracy of our microfluidic rheometer for the measurement of aqueous poly(ethylene oxide) (PEO) solutions. PEO is a standard type of polymer for microfluidic applications, and it has also been previously used for microfluidic rheometry. ${ }^{26,27,49,50}$ We first carried out conventional rheology measurements in order to derive the viscosity curve, represented as shear-viscosity $\eta$ plotted as a function of the shear rate $\dot{\gamma}$ (Figure 1a-e). At a mass concentration $c=0.7 \mathrm{wt} \%$ and at $c=0.63 \mathrm{wt} \%$, the viscosity exhibited a mild shear-thinning behavior above a 
shear-rate value of $\dot{\gamma} \approx 10 \mathrm{~s}^{-1}$. When decreasing the concentration further, the shear thinning became negligible. Regardless of the mass concentration, the shear viscosity decreased monotonically when increasing the temperature, in good agreement with previous findings in the same temperature range. ${ }^{30}$ We then compared the viscosity data obtained using the microfluidic rheometer with those derived via conventional rheometry (Figure 5a-c). For the comparison to be accurate, we compared values of the viscosity evaluated at the same shear rate. The microfluidic rheometer can derive a value of the viscosity at a single shear rate value, depending on the imposed pressure drop. For each PEO concentration and temperature, we evaluated the shear viscosity at a value of the shear-rate such that the shear thinning was negligible (because of the microfluidic rheometer working principle), and we then compared that value with the one obtained using the viscosity curves of Figure 1a-e. The fact that the shear thinning was negligible could be inferred from the particle positions in the microfluidic rheometer: If particles appeared to be aligned, then the shear thinning was negligible. ${ }^{31}$ If they instead appeared to be focused on the walls of the microfluidic channel, then the shear thinning was not negligible, ${ }^{51}$ and the microfluidic rheometer could not be used. ${ }^{26}$ Regardless of the temperature or the polymer concentration, we observed a very good agreement among the data (Figure 5a-b). We also noticed a slight decrease of the viscosity with an increase in the temperature, in line with the experiments using the conventional rheometer.

Despite the good agreement observed between conventional rheometry and microfluidic rheometry data, we wanted to compare our results with existing theoretical predictions for polymer solutions, ${ }^{18}$ to be confident regarding the reliability of the data set as a whole. To this aim, we employed a property called specific viscosity, defined as

$$
\eta_{\mathrm{sp}}=\frac{\eta-\eta_{\mathrm{s}}}{\eta_{\mathrm{s}}}=\frac{\eta}{\eta_{\mathrm{s}}}-1
$$

where $\eta_{s}$ is the solvent viscosity (water in this case). The specific viscosity quantifies the polymer contribution to the solution viscosity, ${ }^{17}$ and it also provides information regarding the molecular conformation. We first noticed that $\eta_{\mathrm{sp}}$ was not changing significantly with the temperature, meaning that the molecular conformation remained substantially unchanged in that temperature range. However, the values of $\eta_{\mathrm{sp}}$ changed with the mass concentration (Figure 5c), in line with previous results. ${ }^{26,27}$ We compared our experimental results with the theoretical scalings for the specific viscosity as a function of temperature, ${ }^{18}$ with $\eta_{\text {sp }} \propto c^{1 / 3 \nu-1}$, where $\nu$ is an exponent that can vary in the range $0.5<\nu<0.6$; its value is related to the polymer conformation. When $\nu=0.5$, the polymer is arranged in a perfect coil where the steric repulsion between monomers is balanced by the solvent-mediated attraction between monomers. ${ }^{17}$ When $\nu>0.5$, the steric repulsion is stronger than the solvent mediated attraction, resulting in a more swelled coil. Our data of $\eta_{\text {sp }}$ versus mass concentration in the range $0.158<c<0.398 \mathrm{wt} \%$ scaled as $\eta_{\mathrm{sp}} \propto c^{1.67}\left(R^{2}=0.98\right)$, before displaying deviations due to the transition from a semidilute unentangled to a semidilute entangled polymer regime, in very good agreement with data presented previously. ${ }^{26}$ From this scaling, we evaluated a value of the exponent $\nu=0.533$, meaning that the polymer assumed a swelled coil configuration that did not change significantly with the temperature. The comparison of the data with the existing theory of polymer solution is essential to provide further reliability of the whole experimental apparatus, and it is also key to assessing the robustness of the longest relaxation time data, as described below.

According to the working principle of the microfluidic rheometer, we could measure the longest relaxation time $\lambda$ simultaneously to the shear viscosity $\eta$ (Figure $5 \mathrm{~d}, \mathrm{e}$ ). We first observed that the values of $\lambda$ were substantially independent of the temperature (Figure $5 \mathrm{~d}$ ), in line with the results on the specific viscosity $\eta_{\mathrm{sp}}$ (Figure $5 \mathrm{~b}$ ). In this case, however, we could not compare the data directly to values from the conventional rheometer, because the rheometer was not sensitive enough to measure such small values of $\lambda$. A loose estimate of the longest relaxation time can be carried out from the viscosity curve as $\lambda=1 / \dot{\gamma}_{\text {cr }}$, where $\dot{\gamma}_{\text {cr }}$ indicates the critical value of shear rate above which the fluid displays shearthinning properties. We then evaluated the value of $\dot{\gamma}_{\mathrm{cr}}=1 / \lambda$, where $\lambda$ was the value of the longest relaxation time calculated using the microfluidic rheometer (dotted lines in Figure 1a-e). We found reasonable agreement between the values of the longest relaxation time measured using the microfluidic rheometer and the onset of the shear-thinning in the viscosity curve of the PEO solutions. Moreover, our data were in good agreement with the theoretical scaling for the longest relaxation time as a function of concentration (Figure $5 \mathrm{e}$ ), being $\lambda \propto c^{2-3 \nu / 3 \nu-1} \propto c^{0.67}\left(R^{2}=0.587\right)$, with $\nu=0.533$ as calculated from the viscosity data, meaning that the data were also in line with theoretical predictions.

In summary, we demonstrated that our microfluidic rheometer could be employed to simultaneously measure the shear viscosity $\eta$ and the longest relaxation time $\lambda$ of several standard PEO solutions with mild or negligible shear-thinning features, and at different imposed temperature values. Data obtained using the microfluidic rheometer were in good agreement with those derived via the bulk rheometer. The data trend with the mass concentration was in agreement with the theoretical predictions for polymers in relatively good solvent, ${ }^{18}$ and with previous experimental data. ${ }^{26}$

4.3. Proof-of-Concept Application Using Hyaluronic Acid Solutions. Hyaluronic acid (HA) is a long macromolecule which is found in vitreous humor, umbilical cord, and joint synovial fluid. ${ }^{16,52}$ In particular, the concentration of hyaluronic acid in synovial fluid is a good indicator of the conditions of the joint. ${ }^{1,53}$ For instance, patients affected by rheumatoid arthritis displayed different concentrations and molecular weights of hyaluronic acid compared to healthy volunteers. ${ }^{54,55}$ The measurement of hyaluronic acid concentration is usually performed using protein assays; ${ }^{54,56}$ however, this involves a long protocol that often can only be performed by specialized staff. We here prove that our microfluidic rheometer can be used to rapidly evaluate different concentrations of HA at different temperatures (an important factor to replicate physiological conditions) via measurement of shear viscosity or longest relaxation time (Figure 6). We followed the same procedure adopted for PEO solutions, and we measured the viscosity curve for the three HA concentrations employed here (in the clinical range for assessing joint quality ${ }^{1}$ ), finding a rheological trend similar to the PEO solutions. We observed that all of the solutions exhibited a mild shear-thinning behavior (Figure 1f-h). During our microfluidic rheometry experiments, we observed particles aligned at the centerline, meaning that the shear- 

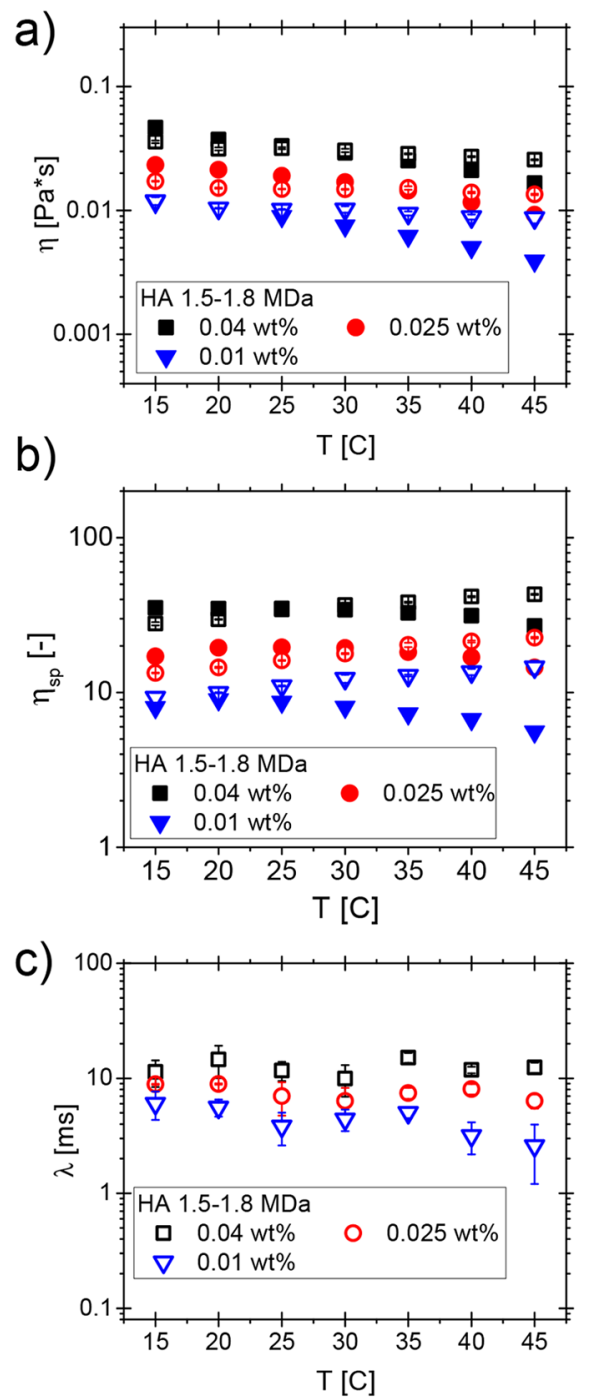

Figure 6. Good agreement is found between conventional rheology and microfluidic rheometry data for hyaluronic acid (HA) solutions. (a) Comparison between conventional rheometry data (closed symbols) and microfluidic rheometry data (open symbols) for the viscosity as a function of temperature, for all of the HA solutions investigated in this work. Values of the viscosity were compared at the same shear rate. Very good agreement was observed for all of the solutions. (b) Same data as in panel a, plotted in terms of specific viscosity $\eta_{\mathrm{sp}}=\eta / \eta_{\mathrm{s}}-1$, where $\eta_{\mathrm{s}}$ is the solvent viscosity (water in this case). (c) Longest relaxation time $\lambda$ measured using the microfluidic rheometer as a function of the temperature $T$ for all of the HA solutions investigated.

thinning properties were negligible. ${ }^{26}$ With this in mind, we compared the viscosity data obtained using the microfluidic rheometer with those from the bulk rheology at the same shear rate value, and we observed very good agreement (Figure 6a). A similar agreement was observed when representing the data in terms of specific viscosity $\eta_{s p}$ (Figure 6b). Similarly to the PEO solutions, we evaluated the longest relaxation time $\lambda$, observing the mild dependence on the temperature, as also observed for PEO solutions (Figure 6c).

An important aspect of our analysis was the fact that, regardless of the temperature, both the shear viscosity $\eta$ and the longest relaxation time $\lambda$ increased monotonically with the concentration, meaning that these rheological parameters could be potentially used as rheo-markers (i.e., rheological biomarkers) to asses the joint quality. While the shear viscosity of hyaluronic acid solutions has long been established as a potential biomarker in the context of joint quality, ${ }^{2}$ to the best of our knowledge, this is the first time that the longest relaxation time is presented as a potential biomarker in the same context. We appreciate that the results reported here represent a proof of concept only, while calling for future studies aimed at establishing whether the microfluidic rheometer can be used as a diagnostic tool.

The shear viscosity has been previously suggested as a potential biomarker for several health conditions and diseases, including general cardiovascular events, ${ }^{6,7}$ stroke, ${ }^{8}$ and periprosthetic joint infection; ${ }^{3}$ however, the longest relaxation time $\lambda$ has never been introduced as a potential biomarker. As already mentioned in the Introduction, the longest relaxation time displays a stronger dependence on the molecular weight than the shear viscosity, ${ }^{18}$ especially in dilute and semidilute unentangled systems, thus making it a potential biomarker to identify the contribution of large macromolecules over smaller ones in solution. In the clinical context, the measurement of $\lambda$ would be a rheomarker superior to the shear viscosity when discrimination between molecules having different molecular weights is essential. For instance, in the context of synovial fluid, this is relevant in the assessment of joint grade ${ }^{1}$ in the context of cardiovascular diseases, an increase of fibrinogen in blood plasma could be better singled out by the contribution of other molecules to its rheological response by measuring $\lambda$ rather than $\eta$, fibrinogen generally having the largest molecular weight among proteins in blood plasma.

Previous investigations could not explore the potential of using $\lambda$ as a biomarker because of technological limitations associated with its measurement with conventional techniques. ${ }^{27,49}$ Each measurement with our microfluidic rheometer required only sample volumes on the order of $100-200 \mu \mathrm{L}$, thus being ideal for the analysis of biological fluids, where sample availability is always a concern. Furthermore, each measurement could be performed in as little as 2 min thanks to the machine-learning-based code for the particle tracking. This means that, in the future, the microfluidic rheometer could offer a valuable alternative to established techniques for rapid diagnostic tests, as well as to monitor disease progression for other diseases or chronic conditions. Please also note that the sensitivity of the microfluidic rheometer toward smaller or larger values of both shear viscosity and longest relaxation time (meaning smaller or larger macromolecule concentration in solution) can be tuned by changing the diameter of the microfluidic device. ${ }^{26,28}$ Specifically, the smaller the channel dimension, the smaller is the value of both $\eta$ and $\lambda$ measurable.

\section{CONCLUSION}

In this work, we introduced a microfluidic rheometer apparatus for rapid measurement of shear viscosity $\eta$ and longest relaxation time $\lambda$ of non-Newtonian solutions, at different temperatures. To the best of our knowledge, this is the first microfluidic platform for the rapid simultaneous measurement of rheological properties of non-Newtonian solutions at different temperatures, using between 100 and $200 \mu \mathrm{L}$ of samples, thus being the ideal platform for the characterizations of biofluids. We first carried out experiments on several poly(ethylene oxide) solutions, finding good agreement between the microfluidic rheometry and the conventional rheometry data. The same data were also in agreement with 


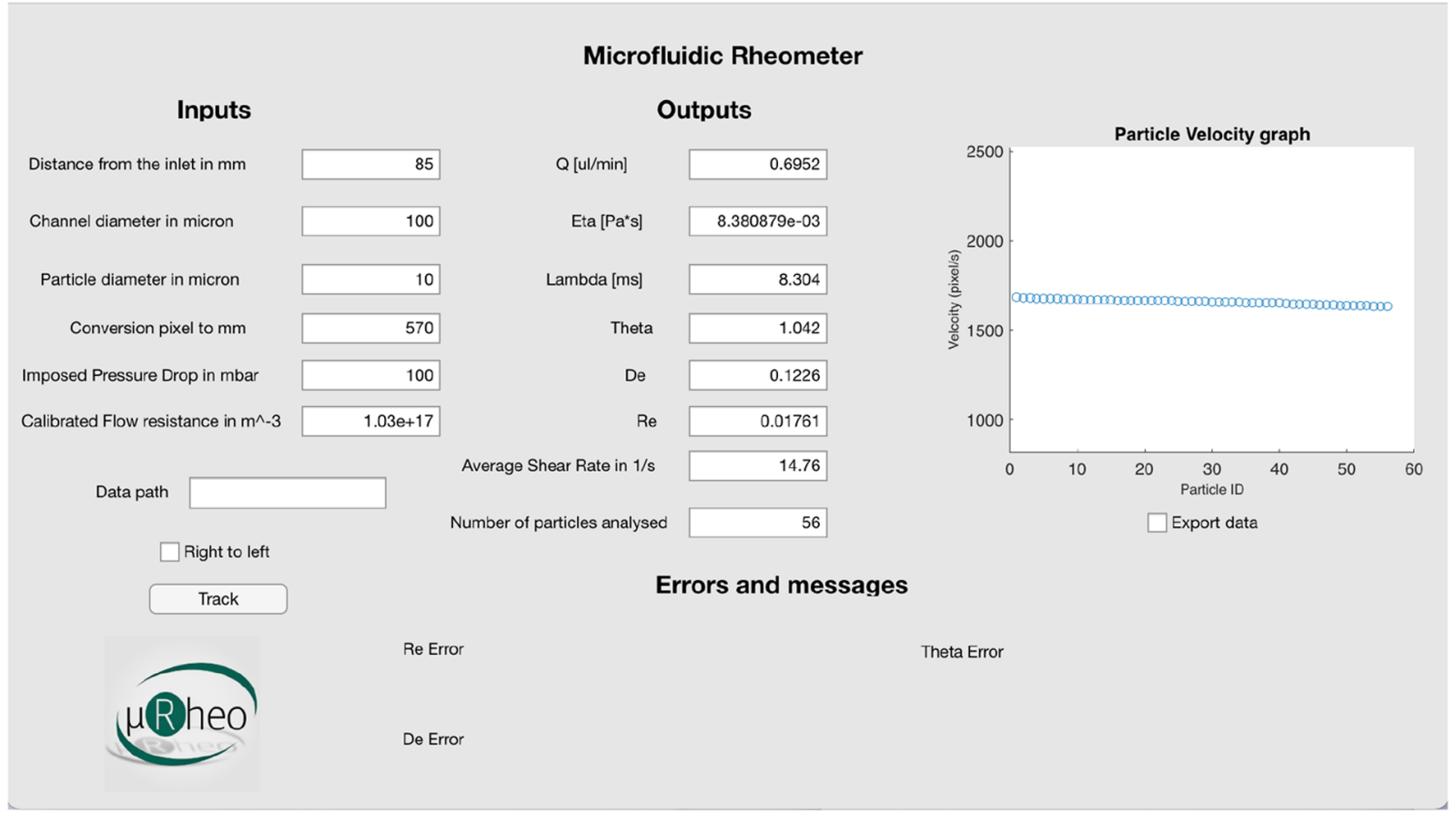

Figure 7. User interface of the Microfluidic Rheometer app employed in this study. The input parameters are reported on the left and the output parameters in the center, while the velocity of each particle expressed in pixels per second is reported on the right. Error messages are displayed when the theoretical assumptions underlying eq 2 are not met (see full text for details).

existing theoretical predictions for polymers in relatively good solvent. ${ }^{18}$ We then employed the same apparatus to evaluate the values of $\eta$ and $\lambda$ for three hyaluronic acid solutions at concentrations falling in the clinical range for joint quality. Regardless of the imposed temperature, we observed a clear trend for both $\eta$ and $\lambda$ with the concentration, thus speculating about the possibility of using our microfluidic rheometer in the clinical context. Future works will have to investigate whether our speculations stand, as well as to explore whether the longest relaxation time $\lambda$ can actually be used as a potential biomarker for diagnostic purposes or to monitor disease progression.

In terms of machine learning, it is important to note that our first assumption, the simplicity of the particles trajectory, will only apply in the case of a few applications. Future works targeting successful analysis of more complex trajectories through a hybrid approach may enable further microfluidic applications. A key criticism of many of the current available tools is that they are often trained and tested on videos taken using the same experimental apparatus, rather than looking at the possibility of using the same algorithm on different experimental apparati. ${ }^{39}$ Our algorithms were trained and tested on images from the same experimental setup, containing minimal artifacts and background noise. Some artificial poor quality was tested by adjustment of the exposure time, and our algorithm successfully dealt with this variation. However, this is an area that warrants further validation and testing. It is important to note, however, that through the employment of a neural network to predict key parameters based on the initial frames of a video, we propose a flexible solution which we feel will deal with these types of challenges well in future applications.

\section{APPENDIX: MICROFLUIDIC RHEOMETER APP USER INTERFACE}

We employed Matlab to prepare a user-friendly application, called $\mu$ Rheo, that can be used on the avi videos recorded using our digital microscope (Figure 7). The user is first asked to insert some parameters including the distance from the inlet $L_{z}$, the channel diameter $D$, the particle diameter $d_{\mathrm{p}}$, the imposed pressure drop $\Delta P$, and the calibrated flow resistance $\mathcal{R}$. Another important input parameter is the one labeled "Conversion pixel to $\mathrm{mm}$ ", which is a value representing how many pixels are included in $1 \mathrm{~mm}$; in our case, each millimeter was made of 570 pixels. This value is important, as it is required to convert pixel size in metric units; also, this parameter depends on the microscope magnification and on the camera magnification, if any type of adapter is used to connect the camera to the microscope. In our case, the camera was embedded in the digital microscope, and the only variable was the magnification which was kept constant for all of the experiments. The user can then specify the path where temporary data can be stored ("Data path" entry) and then press the button "Track"; this will open the windows where the file is to be selected. Upon selection, the machine-learningaided particle tracking step begins. At the end of it, velocities are plotted in the graph on the left as particle velocity versus particle ID, and they are also ordered in descending order. With reference to Figure 7, particle velocity was nearly constant, meaning that all of the particles were at the centerline. The user is then tasked to select the maximum velocity, which will then be used to evaluate the volumetric flow rate $Q$ the viscosity $\eta$ via eq 1 , and the longest relaxation time $\lambda$ via eq 2 . Upon completion, the output parameters will be displayed in the column labeled "outputs". Any error message related to the underlying assumptions of the model employed to evaluate $\lambda$ via eq 2 is displayed on the bottom in 
section "Error and messages". Finally, the user can export the data by clicking the "Export data" button. Data will be saved in two different categories: 'outputs.txt' contains the output parameters, while 'velocity.txt' contains the list of velocities for each particle.

Original video files and the Microfluidic Rheometer application installer for Windows together with the original Matlab code are available upon reasonable request made to the corresponding author.

\section{AUTHOR INFORMATION}

\section{Corresponding Author}

Francesco Del Giudice - Department of Chemical Engineering, Faculty of Science and Engineering, School of Engineering and Applied Science, Swansea University Fabian Way, Swansea SA1 8EN, United Kingdom; (1) orcid.org/ 0000-0002-9414-6937; Email: francesco.delgiudice@ swansea.ac.uk

\section{Author}

Claire Barnes - Department of Biomedical Engineering, Faculty of Science and Engineering, School of Engineering and Applied Science, Swansea University Fabian Way, Swansea SA1 8EN, United Kingdom

Complete contact information is available at: https://pubs.acs.org/10.1021/acs.analchem.1c05208

\section{Author Contributions}

F.D.G. designed the study, performed the measurements, and analyzed the data. C.B. wrote the Matlab code and trained the neural network. Both authors designed the application GUI. F.D.G. and C.B. wrote and approved the manuscript.

\section{Notes}

The authors declare no competing financial interest.

\section{ACKNOWLEDGMENTS}

F.D.G. acknowledges support from EPSRC New Investigator Award (grant ref no. EP/S036490/1). F.D.G. thanks Dr. Rebecca Hudson for the help provided in setting up the conventional rheometer. The authors thank Dr. Shivani Sathish from the Okinawa Institute of Science and Technology for helpful comments on the manuscript. This research contributes to the IMPACT operation which has been part-funded by the European Regional Development Fund through the Welsh Government and Swansea University.

\section{REFERENCES}

(1) Temple-Wong, M. M.; Ren, S.; Quach, P.; Hansen, B. C.; Chen, A. C.; Hasegawa, A.; D’Lima, D. D.; Koziol, J.; Masuda, K.; Lotz, M. K.; Sah, R. L. Arthritis research \& therapy 2016, 18, 1-8.

(2) Ragan, C.; Meyer, K. J. Clin. Invest. 1949, 28, 56-59.

(3) Fu, J.; Ni, M.; Chai, W.; Li, X.; Hao, L.; Chen, J. Journal of arthroplasty 2019, 34, 1197-1200.

(4) Chang, X.; Yamada, R.; Yamamoto, K. Arthritis Res. Ther 2005, 7, R268.

(5) Guo, Y.; Wei, T.; Hu, N.; Zhou, X. Immunologic Research 2021, $1-10$.

(6) Lowe, G.; Lee, A.; Rumley, A.; Price, J.; Fowkes, F. British journal of haematology 1997, 96, 168-173.

(7) Jeong, S.-K.; Cho, Y. I.; Duey, M.; Rosenson, R. S. Cardiovascular drugs and therapy 2010, 24, 151-160.

(8) Rasyid, A.; Harris, S.; Kurniawan, M.; Mesiano, T.; Hidayat, R. Annals of Neurosciences 2019, 26, 30-34.

(9) Bateman, R. J.; et al. N Engl J. Med. 2012, 367, 795-804.
(10) Buss, H.; Chan, T. P.; Sluis, K. B.; Domigan, N. M.; Winterbourn, C. C. Free Radical Biol. Med. 1997, 23, 361-366.

(11) Sapan, C. V.; Lundblad, R. L. Prot. Clin. Appl. 2015, 9, 268276.

(12) Sathish, S.; Shen, A. Q. JACS Au 2021, 1, 1815.

(13) Brust, M.; Schaefer, C.; Doerr, R.; Pan, L.; Garcia, M.; Arratia, P.; Wagner, C. Physical review letters 2013, 110, 078305.

(14) Kim, B.; Lee, S. S.; Yoo, T. H.; Kim, J. M. Electrophoresis 2021, 42, 2238.

(15) Haward, S. J.; Odell, J. A.; Berry, M.; Hall, T. Rheologica acta 2011, 50, 869-879.

(16) Haward, S. J. Biopolymers 2014, 101, 287-305.

(17) Rubinstein, M.; Colby, R. H. Polymer Physics; Oxford University Press: New York, 2003; Vol. 23.

(18) Colby, R. H. Rheologica acta 2010, 49, 425-442.

(19) Sharma, V.; Jaishankar, A.; Wang, Y.-C.; McKinley, G. H. Soft Matter 2011, 7, 5150-5160.

(20) Gupta, S.; Wang, W. S.; Vanapalli, S. A. Biomicrofluidics 2016, 10,043402 .

(21) Del Giudice, F. Micromachines 2022, 13, 167.

(22) Choi, S.; Park, J.-K. Small 2010, 6, 1306-1310.

(23) Arosio, P.; Hu, K.; Aprile, F. A.; Müller, T.; Knowles, T. P. Analytical chemistry 2016, 88, 3488-3493.

(24) Lee, T.-A.; Liao, W.-H.; Wu, Y.-F.; Chen, Y.-L.; Tung, Y.-C. Analytical chemistry 2018, 90, 2317-2325.

(25) Kang, H.; Jang, I.; Song, S.; Bae, S.-C. Analytical chemistry 2019, 91, 4868-4875.

(26) Del Giudice, F. Phys. Fluids 2020, 32, 052001.

(27) Del Giudice, F.; D’Avino, G.; Greco, F.; De Santo, I.; Netti, P. A.; Maffettone, P. L. Lab Chip 2015, 15, 783-792.

(28) Del Giudice, F.; Calcagno, V.; Esposito Taliento, V.; Greco, F.; Netti, P. A.; Maffettone, P. L. J. Rheol. 2017, 61, 13-21.

(29) Macosko, C. W. Rheology: Principles, Measurements, and Applications; Wiley-VCH, 1994.

(30) Bahlouli, M. I.; Bekkour, K.; Benchabane, A.; Hemar, Y.; Nemdili, A. Appl. Rheol. 2013, 23, 13435-1.

(31) Zhou, J.; Papautsky, I. Microsystems \& Nanoengineering 2020, 6, $1-24$.

(32) D’Avino, G.; Greco, F.; Maffettone, P. L. Annu. Rev. Fluid Mech. 2017, 49, 341-360.

(33) Lu, X.; Liu, C.; Hu, G.; Xuan, X. J. Colloid Interface Sci. 2017, 500, 182-201.

(34) Bird, R. B.; Stewart, W. E.; Lightfoot, E. N. Transport Phenomena; John Wiley \& Sons, 2006; Vol. 1.

(35) Leshansky, A. M.; Bransky, A.; Korin, N.; Dinnar, U. Physical review letters 2007, 98, 234501.

(36) Xia, H.; Wu, J.; Zheng, J.; Zhang, J.; Wang, Z. Lab Chip 2021, $21,1241-1268$.

(37) Higdon, J.; Muldowney, G. J. Fluid Mech. 1995, 298, 193-210.

(38) Romeo, G.; D’Avino, G.; Greco, F.; Netti, P. A.; Maffettone, P. L. Lab Chip 2013, 13, 2802-2807.

(39) Cheng, H.-J.; Hsu, C.-H.; Hung, C.-L.; Lin, C.-Y. A review for cell and particle tracking on microscopy images using algorithms and deep learning technologies. Biomedical Journal 2021, DOI: 10.1016/ j.bj.2021.10.001.

(40) Yao, Y.; Smal, I.; Grigoriev, I.; Akhmanova, A.; Meijering, E. Bioinformatics 2020, 36, 4935-4941.

(41) Arts, M.; Smal, I.; Paul, M. W.; Wyman, C.; Meijering, E. Sci. Rep. 2019, 9, 1-10.

(42) Yang, L.; Qiu, Z.; Greenaway, A. H.; Lu, W. IEEE transactions on biomedical engineering 2012, 59, 2040-2050.

(43) Jaqaman, K.; Loerke, D.; Mettlen, M.; Kuwata, H.; Grinstein, S.; Schmid, S. L.; Danuser, G. Nat. Methods 2008, 5, 695-702.

(44) Tsai, H.-F.; Gajda, J.; Sloan, T. F.; Rares, A.; Shen, A. Q. SoftwareX 2019, 9, 230-237.

(45) Hu, T.; Xu, S.; Wei, L.; Zhang, X.; Wang, X. Bioinformatics 2021, 37, 285-287. 
(46) Girshick, R.; Donahue, J.; Darrell, T.; Malik, J. Proceedings of the IEEE conference on computer vision and pattern recognition 2014, 580587.

(47) Otsu, N. IEEE transactions on systems, man, and cybernetics 1979, 9, 62-66.

(48) Crocker, J. C.; Grier, D. G. J. Colloid Interface Sci. 1996, 179, 298-310.

(49) Zilz, J.; Schäfer, C.; Wagner, C.; Poole, R. J.; Alves, M. A.; Lindner, A. Lab Chip 2014, 14, 351-358.

(50) Gupta, S.; Vanapalli, S. A. Phys. Fluids 2020, 32, 012006.

(51) Del Giudice, F.; Sathish, S.; D’Avino, G.; Shen, A. Q. Analytical chemistry 2017, 89, 13146-13159.

(52) Kogan, G.; Soltés, L.; Stern, R.; Gemeiner, P. Biotechnology letters 2006, 29, 17-25.

(53) Migliore, A.; Procopio, S. Clinical Cases in Mineral and Bone Metabolism 2015, 12, 31.

(54) Sasaki, Y.; Uzuki, M.; Nohmi, K.; Kitagawa, H.; Kamataki, A.; Komagamine, M.; Murakami, K.; Sawai, T. International journal of rheumatic diseases 2011, 14, 313-319.

(55) Emlen, W.; Niebur, J.; Flanders, G.; Rutledge, J. J. Rheumatology 1996, 23, 974-978.

(56) Majeed, M.; McQueen, F.; Yeoman, S.; McLean, L. Annals of the rheumatic diseases 2004, 63, 1166-1168.

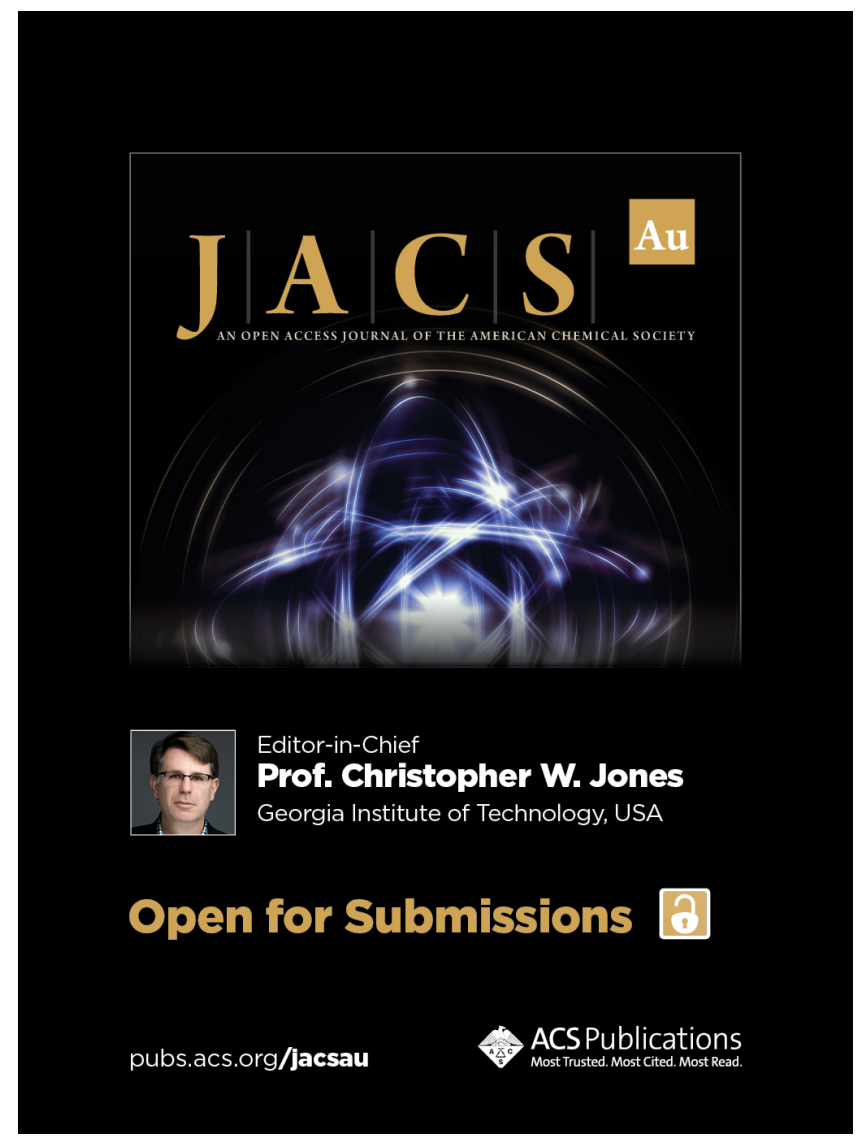

